\title{
A influência da quiropraxia na dor cervical de origem postural
}

\author{
The influence of chiropractic care on cervical pain of postural \\ La influencia de la quiropraxia en el dolor cervical de origen postural
}

Recebido: 06/11/2021 | Revisado: 11/12/2021 | Aceito: 12/12/2021 | Publicado: 20/12/2021

\author{
Jaini Ribeiro Lima \\ ORCID: https://orcid.org/0000-0001-6977-4424 \\ Instituto Esperança de Ensino Superior, Brasil \\ E-mail: jainiribeiro02@gmail.com \\ Sara Maria Azevedo de Sousa \\ ORCID: https://orcid.org/0000-0001-5116-9314 \\ Instituto Esperança de Ensino Superior, Brasil \\ E-mail: sarasousa20545@gmail.com \\ Yamara Rodrigues da Costa \\ ORCID: https://orcid.org/0000-0001-9287-1089 \\ Instituto Esperança de Ensino Superior, Brasil \\ E-mail: yamaracosta10@gmail.com \\ Jorge Carlos Menezes Nascimento Junior \\ ORCID: https://orcid.org/0000-0003-2593-7804 \\ Instituto Esperança de Ensino Superior, Brasil \\ E-mail: jcmnj@hotmail.com
}

\begin{abstract}
Resumo
Objetivo: O objetivo dessa pesquisa é determinar a influência da quiropraxia na dor cervical de origem postural em estudantes de uma Instituição de Ensino Superior. Métodos: Trata-se de uma pesquisa de campo com aplicação de ficha de avaliação para coleta de dados e aplicação da técnica de quiropraxia. A aplicação da ficha de avaliação constava com tópicos como: identificação, testes especiais para a cervical, seguida da aplicação da escala de EVA no início e após cada sessão avaliando assim a dor do paciente, totalizando quatro sessões ao todo, com duração de 20-30 minutos, e com intervalo de 7 a 10 dias entre as sessões. Resultados: De maneira geral, a quiropraxia apresenta resultados significativos para o tratamento da dor cervical, obtendo melhor eficácia principalmente após as quatro sessões, porém novos estudos são necessários para melhor evidenciar o potencial da técnica.
\end{abstract}

Palavras-chave: Dor cervical; Fisioterapia; Quiropraxia.

\begin{abstract}
Objective: The aim of this research is to determine the influence of chiropractic on cervical pain of postural origin in students of a Higher Education Institution. Methods: This is a field research with application of an evaluation form for data collection and application of the chiropractic technique. The application of the evaluation form consisted of topics such as: identification, special tests for cervical, followed by the application of the VAS scale at the beginning and after each session thus evaluating the patient's pain, totaling four sessions in all, lasting 20-30 minutes, and with an interval of 7 to 10 days between sessions. Results: In general, chiropractic presents significant results for the treatment of cervical pain, obtaining better efficacy mainly after the four sessions, but further studies are needed to better highlight the potential of the technique.
\end{abstract}

Keywords: Cervical pain; Physiotherapy; Chiropractic.

\section{Resumen}

Objetivo: El objetivo de esta investigación es determinar la influencia de la quiropráctica en el dolor cervical de origen postural en estudiantes de una Institución de Educación Superior. Métodos: Se trata de una investigación de campo con aplicación de un formulario de evaluación para la recolección de datos y aplicación de la técnica quiropráctica. La aplicación del formulario de evaluación consistió en temas como: identificación, pruebas especiales para cervicales, seguido de la aplicación de la escala VAS al inicio y después de cada sesión evaluando así el dolor del paciente, totalizando cuatro sesiones en total, con una duración de 20-30 minutos, y con un intervalo de 7 a 10 días entre sesiones. Resultados: En general, la quiropráctica presenta resultados significativos para el tratamiento del dolor cervical, obteniendo una mejor eficacia principalmente después de las cuatro sesiones, pero se necesitan más estudios para resaltar mejor el potencial de la técnica.

Palabras clave: Dolor cervical; Fisioterapia; Quiropráctica. 


\section{Introdução}

As cervicalgias são comuns em diversas faixas etárias em ambos os sexos, possuindo elevada predominância nas síndromes dolorosas corporais, sendo a segunda maior causa de dor na coluna vertebral, perdendo apenas para a dor lombar (Silva et al., 2012; Dalla, 2018). Acomete um número considerável de indivíduos, com média de $12 \%$ a $34 \%$ da população adulta em alguma fase da vida, com maior incidência no sexo feminino, trazendo prejuízos nas suas atividades de vida diária (Gardin \& Felipe, 2013). Esta doença pode estar relacionada a diferentes causas como por exemplo, movimentos bruscos, longa permanência em posição inadequada, esforço ou trauma, raramente se inicia de maneira súbita (Galera et al., 2017).

Pessoas com dor na coluna cervical adquirem uma postura de defesa, com rigidez na movimentação, o que faz alterar a mobilidade da articulação envolvida. Se formos realizar uma palpação, o paciente irá referir dor e sensação de fadiga muscular (Bacchi et al., 2013). O membro superior é normalmente afetado com alteração na sensibilidade e diminuição da força muscular (Silva \& Barbosa, 2021).

Dentre os tratamentos para cervicalgia, a quiropraxia, uma profissão na área da saúde que se dedica ao diagnóstico, tratamento e prevenção de alterações do sistema músculo-esquelético e seus efeitos sobre a função do sistema nervoso, que enfatiza terapias manuais, tem sido indicada, pois ajuda a restaurar a relação e função articulares normais, restabelecendo a integridade neurológica, influenciando os processos fisiológicos, principalmente em casos de hipersecreção e disfunções neuromusculares (Maia \& de Freitas, 2012).

A quiropraxia na região cervical apresenta resultados ótimos, pois quando uma vértebra não se articula bem com a outra, acaba limitando seus movimentos, ocorrendo assim uma desarmonia no conjunto todo. Com a quiropraxia é possível restabelecer a harmonia vertebral, removendo assim o que está causando irritação nervosa, melhorando seus movimentos, a circulação, reduzindo edema e a dor, fazendo com que o corpo volte a ser capaz de fazer o que ele foi projetado para realizar (Esteves et al., 2013; da Silva et al., 2016).

Com isso, o objetivo deste estudo é identificar a influência do ajuste quiroprático na dor cervical de origem postural em estudantes de uma Instituição de Ensino Superior.

\section{Metodologia}

O presente estudo tratou-se de uma pesquisa quantitativa, que remete-se ao uso da quantificação de dados e informações apurados durante a coleta e no tratamento (Diehl \& Tatim, 2004; Proetti, 2018); de caráter descritivo, pois buscou descobrir com precisão características de determinada população ou fenômeno, e o estabelecimento de relações entre as variáveis (Danton, 2002); de cronologia transversal que analisou dados em um tempo definido, examinando a relação entre variáveis de interesse; prospectivo por se tratar de um estudo que apresente exigências inerentes a padronização e qualidade das informações colhidas (Fletcher, 2021).

Esta pesquisa ocorreu nos meses de setembro a novembro de 2021, após à aprovação do Comitê de Ética em Pesquisa CAAE: 45248521.0.0000.5168 e liberação do Instituto Esperança de Ensino Superior - IESPES.

A pesquisa contou com 18 participantes, que foram atendidos uma vez por semana ou a cada 10 dias, com direito há 4 sessões de atendimentos por pessoa. A forma de captação desses indivíduos se deu por meio de convite colocado no mural informativo do IESPES e no perfil oficial do Instagram dos autores. Foram incluídos na pesquisa participantes maiores de idade, que estivessem regularmente matrículados no Instituto Esperança de Ensino Superior, que apresentassem dor cervical a pelo menos 1 mês, que utilizassem smartphone há pelo menos 1 ano e que não possuissem comorbidades (diabéticos, hipertensos) ou qualquer vínculo trabalhista formal ou informal. 
Inicialmente foi realizada a identificação dos participantes da pesquisa, que foram selecionados de acordo com critérios de inclusão e exclusão. Os dados coletados foram armazenados em uma ficha criada pelos autores, contendo itens como: codinome dos participantes, data da coleta, gênero, sinais e sintomas, queixas essas que foram avaliados através da escala de dor (EVA), e 4 testes especiais (teste de compressão, teste de tração, teste de valsava e teste dix-hallpike).

Para iniciar a pesquisa, os (as) participantes foram levados para uma sala reservada, com macas, temperatura confortável, onde a técnica de ajuste quiroprático seria aplicada. Antes da realização da técnica foi explicado ao participante o que seria feito antes, durante e após a realização da técnica; foi esclarecido também que seria feito uma manobra na região cervical do participante em baixa amplitude e alta velocidade, onde iria sentir e ouvir um "estalo", chamado cavitação e faz parte da técnica, isso significa que foram liberados os gases (co2) que estavam armazenados na cápsula articular. Em seguida foi aplicada a escala de dor EVA e aplicado também quatro testes voltados para região cervical, afim de descartar prováveis dores de origem neuropáticas, os participantes que apresentassem testes positivos seriam excluídos. Após esses procedimentos, dava-se início à aplicação da técnica que consistia em realizar uma tração seguida de uma inclinação lateral do pescoço e de uma rotação com alta velocidade e baixa amplitude na região cervical nos ângulos corretos, seguindo todas a recomendações necessárias sobre a técnica proposta na pesquisa.

Após a realização da técnica, aplicavasse novamente a escala de dor para que se comparasse se houve ou não melhora dos sintomas descritos pelos participantes. A coleta dos dados foi realizada com o máximo de clareza das informações coletadas, buscando proporcionar maior veracidade a pesquisa.

As análises dos dados foram realizadas utilizando o software GraphPad Prism versão 9.0. Foram realizados testes de normalidades, que indicou uma distribuição Gaussiana dos dados, e a partir dessa definição, testes paramétricos foram adotados. Para a análise comparando as sessões como um todo (intragrupo) foi realizado o teste One way ANOVA. Para a comparação entre sessões, utilizou-se o teste de Turkey com medidas repetidas, ajustado para medidas mistas, visto que alguns participantes não responderam a escala de dor em todas as sessões.

Os dados estão apresentados em formato de tabelas e gráficos e foi adotado o valor de $\mathrm{p}<0,05$ para diferenças estatísticamente significativas, com intervalo de confiança de $95 \%$.

\section{Resultados}

A pesquisa foi composta por pacientes que se dispuseram a participar voluntariamente do estudo e procuraram atendimento quiroprático, com queixa álgica na região cervical.

Desta forma, a amostra constou com 18 participantes de ambos os sexos, predominância para o sexo feminino (89\%) sobre o sexo masculino (11\%), com idade entre 19 a 50 anos, todos atendidos no loboratório de avaliação fisioterapêutica, localizado no Campus II do IESPES - Santarém-Pará.

$\mathrm{Na}$ análise comparativa entre a escala EVA nas 4 sessões, observa-se que os valores diminuíram conforme os números de sessões, uma vez que na sessão 1 o nível de dor variou entre 2 a 7, na sessão 2 o nível de dor reduziu, variando de 1 a 6 , na sessão 3 houve uma redução ainda melhor ( 1 a 3 ) e na sessão 4 reduziu significamente variando de 0 a 2 . Essas diferenças ao longo das sessões na análise estatística indicaram redução significativa da dor $(\mathrm{p}<0,0001)$, uma vez que o valor de $\mathrm{p}$ é considerado importante quando for $<0,05$ (Figura 1). 
Figura 1 - Comparação da escala EVA entre as 4 sessões.

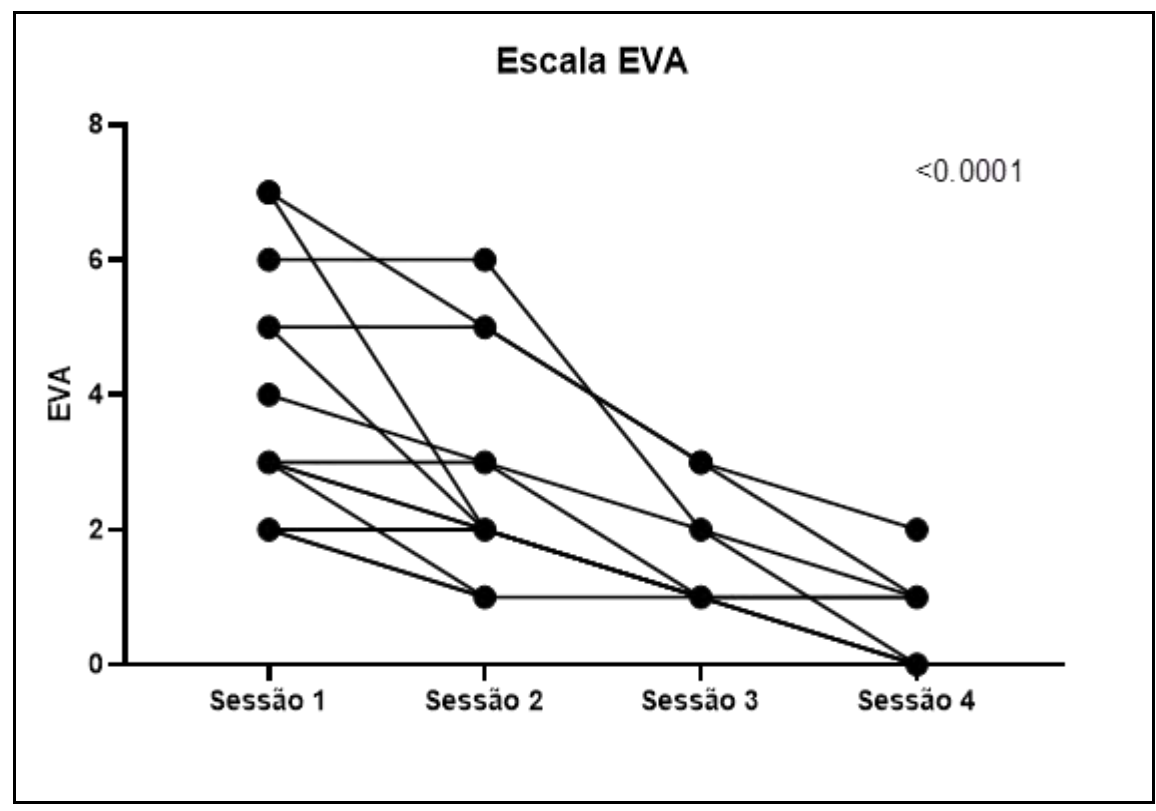

Fonte: Autores (2021).

A análise descritiva dos valores de dor reportados pelos sujeitos utilizando a escala EVA está detalhada na Tabela 1. A média de dor avaliada pela escala EVA foi obtida através da soma do nível de dor relatada pelos participante e dividido pelo número de participantes em cada sessão.

Tabela 1 - Análise descritiva da escala de dor pela EVA de acordo com as sessões.

\begin{tabular}{|c|c|c|c|c|}
\hline $\begin{array}{c}\text { Análise descritiva } \\
\text { EVA }\end{array}$ & Sessão 1 & Sessão 2 & Sessão 3 & Sessão 4 \\
\hline Número de respostas & 18 & 13 & 10 & 9 \\
\hline Média & 3,611 & 2,692 & 1,600 & 0,6667 \\
\hline Desvio padrão & 1,720 & 1,653 & 0,8433 & 0,7071 \\
\hline Mínimo & 2,000 & 1,000 & 1,000 & 0,000 \\
\hline Máximo & 7,000 & 6,000 & 3,000 & 2,000 \\
\hline
\end{tabular}

Fonte: Autores (2021).

Na comparação entre as sessões, foi possível identificar o mesmo padrão de redução da dor mensurada pela escala EVA, como demonstra a Figura 2, que ilustra a diferença média entre cada sessão. 
Figura 2 - Comparação da escala EVA entre todas as 4 sessões.

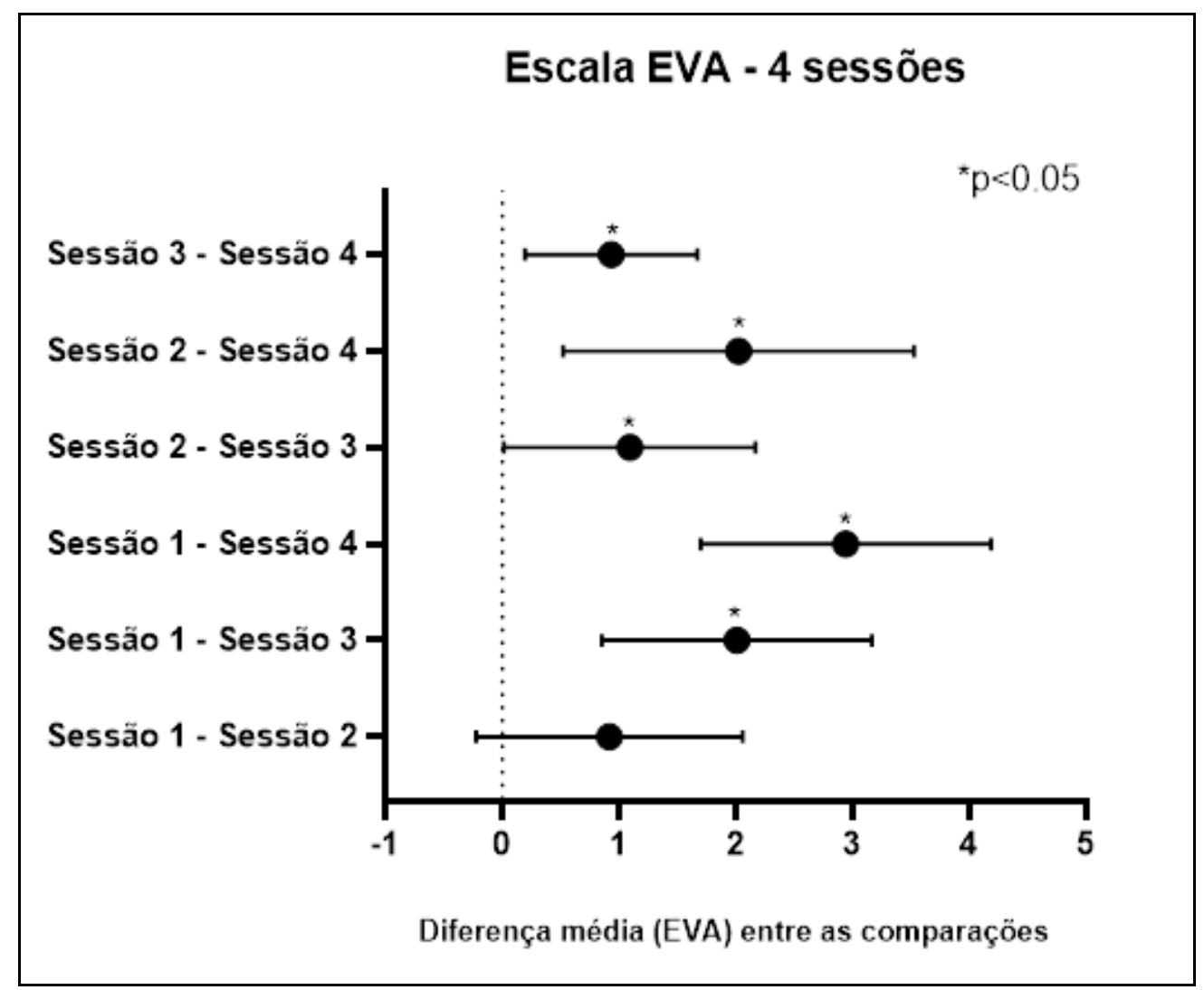

Fonte: Autores (2021).

Observa-se que na comparação dentre as diferenças médias na escala de dor EVA entre as sessões, a menor diferença estatisticamente significativa foi encontrada comparando as sessões 3 e 4 (1,6 vs 0,6; diferença média de 0,9; p<0,01) e a maior diferença estatisticamente significativa foi encontrada comparando as sessões 1 e 4 (3,6 vs 0,6; diferença média de 2,9; p<0,0003), como demonstra a Tabela 2. Mesmo apresentando redução de 0,9 pontos na escala EVA, não houve diferença estatisticamente significativa na comparação entre as sessões 1 e $2(p=0,13)$.

Tabela 2 - Comparações multiplas da escala EVA entre as 4 sessões.

\begin{tabular}{|c|c|c|c}
\hline $\begin{array}{c}\text { Teste de Tukey } \\
\text { (multiplas comparações) }\end{array}$ & $\begin{array}{c}\text { Diferença } \\
\text { média }\end{array}$ & IC (95\%) & Valor de p \\
\hline \#Sessão 1 vs. Sessão 2 & 0,9 & $-0,2217-2,059$ & 0,1317 \\
\hline Sessão 1 vs. Sessão 3 & 2,0 & $0,8535-3,169$ & 0,0019 \\
\hline Sessão 1 vs. Sessão 4 & 2,9 & $1,701-4,188$ & 0,0003 \\
\hline Sessão 2 vs. Sessão 3 & 1,1 & $0,01295-2,172$ & 0,0473 \\
\hline Sessão 2 vs. Sessão 4 & 2,0 & $0,5224-3,529$ & 0,0110 \\
\hline Sessão 3 vs. Sessão 4 & 0,9 & $0,1940-1,673$ & 0,0157 \\
\hline
\end{tabular}

\#Não houve diferença estatisticamente significativa $(p>0,05)$ na comparação pelo teste de Turkey; IC - intervalo de confiança. Fonte: Autores (2021)

\section{Discussão}

Diante do exposto, pode-se dizer que a dor apresentada pelos participantes diminuiu expressivamente, uma vez que a intensidade de dor que os pacientes relataram através da EVA, antes de iniciar o tratamento variava entre 2-7, e após o tratamento foi de 3-0. Tais resultados condizem com o estudo feito por Gama et al. (2019), que utilizou a quiropraxia para o 
Research, Society and Development, v. 10, n. 17, e32101724379, 2021

CC BY 4.0) | ISSN 2525-3409 | DOI: http://dx.doi.org/10.33448/rsd-v10i17.24379

tratamento de cervicalgia com 10 pacientes e constataram a média 7 de intensidade de dor na Escala Visual Analógica antes do início do tratamento, e ao final do tratamento média 3 na EVA.

Bracht (2019) em seu estudo composto por 90 indivíduos que foram divididos em dois grupos (manipulação vertebral x sham), concluiu que os participantes do grupo que receberam a manipulação vertebral apresentaram uma melhora clínica superior aos participantes do grupo controle (sham). E os dados coletados durante o estudo confirmam e estendem os achados da literatura e reforçam que a terapia manipulativa apresenta benefícios para pacientes com cervicalgia.

No estudo feito por dos Santos Pacheco et al. (2018), no qual foram revisados 30 artigos científicos, fala que a utilização da quiropraxia no tratamento da cervicalgia mostrou resultados satisfatórios, havendo aumento da amplitude de movimento da coluna cervical, porém sugere-se a realização de novos trabalhos, com um maior número de ensaios clínicos controlados e aleatórios envolvendo a quiropraxia e analisando concomitantemente a amplitude de movimento de outras regiões como torácica e lombar a fim de constatar de forma eficaz os benefícios do tratamento da quiropraxia em pacientes com cervicalgia, bem como a utilização de métodos de avaliação mais fidedignos, com intuito de comprovar os seus reais efeitos no tratamento da cervicalgia.

Para de Barros et al. (2020), em seu estudo com 40 voluntários que foram randomizados em quatro grupos que receberam uma única intervenção: G1: manipulação quiroprática; G2: Controle; G3: TENS e G4: TENS + manipulação quiroprática. Os resultados demonstram que houve uma redução significativa da dor no G1 em comparação ao G2 (p <0,0001), o tratamento quiroprático com e sem TENS foi eficaz no aumento dos itens de ADM e NDI (Índice de Incapacidade Pescoço); já em relação a combinação de terapias não resultou em ganhos no manejo da cervicalgia comparados aos tratamentos unimodais.

Gorrell et al. (2016) em seu estudo com 65 participantes, demonstra que uma única manipulação cervical é capaz de produzir benefícios imediatos e de curto prazo para dor mecânica no pescoço (MNP). O estudo também demonstra que nem todas as técnicas manipuladoras têm o mesmo efeito. Os resultados relatados neste estudo são consistentes com a hipótese de que as características biomecânicas de diferentes técnicas de manipulação da coluna vertebral podem ser responsáveis por efeitos clínicos variados. No entanto, os resultados não são definitivos, e novas pesquisas que investigam a natureza dessas mudanças são justificadas.

Na revisão bibliográfica realizada por Barros et al. (2020), foram utilizados 12 artigos científicos, dois sobre a quiropraxia e o restante sobre acupuntura, como método para o tratamento de patologias da coluna vertebral, ao final do estudo ele relata que as duas técnicas mostram-se eficientes e seguras tanto para a redução do alívio da dor, quanto para a melhoria da incapacidade e mobilidade. Porém, no que se refere ao tratamento quiroprático, seus melhores resultados são observados quando associados a outros tratamentos.

\section{Conclusão}

A dor cervical é ocasionada na maioria das vezes por alterações mecânicos-posturais, dentre as diferentes técnicas utilizadas por fisioterapeutas para o tratamento, a quiropraxia têm se tornado bastante comum, uma vez que vem apresentando bons resultados apesar de poucos estudos científicos.

A presente pesquisa verificou que houve melhora da dor cervical nos pacientes após ajuste quiroprático. Os resultados demonstram que há alívio imediato da sintomatologia dolorosa nessa região após a primeira sessão, porém melhores resultados são observados entre a 1-4 sessão.

Portanto, conforme os resultados apresentados por essa pesquisa, mostra-se que poucas sessões de quiropráxia são eficazes para alcançar um resultado satisfatório na redução da cervicalgia, porém, é necessário a realização de novas pesquisas 
Research, Society and Development, v. 10, n. 17, e32101724379, 2021

CC BY 4.0) | ISSN 2525-3409 | DOI: http://dx.doi.org/10.33448/rsd-v10i17.24379

afim de melhor evidenciar os benefícios dessa terapia.

\section{Referências}

Bacchi, C. D. A., Candotti, C. T., Noll, M., \& Minossi, C. E. D. S. (2013). Avaliação da qualidade de vida, da dor nas costas, da funcionalidade e de alterações da coluna vertebral de estudantes de fisioterapia. Motriz: Revista de Educação Física, 19(2), 243-251.

Barros, J. N., Leite, C. L., \& Perreira, D. M. S. (2020). A acupuntura e quiropraxia no tratamento das patologias da coluna vertebral. Revista Científica Multidisciplinar Núcleo do Conhecimento, 9, 47-60.

Bracht, M. A. (2019). Efeitos da manipulação vertebral e educação em dor na cervicalgia crônica: estudo clínico randomizado e controlado.

Byfield, D. (2011). Technique Skills in Chiropractic E-book. Elsevier Health Sciences.

da Silva, G. C. A., de Almeida Chiapetta, C., Lopes, A. C. B. S., \& Barbosa, F. K. (2016). Quiropraxia: Revisão Sistemática. UNILUS Ensino e Pesquisa, 13(30), 224.

Dalla Pasqua, T. P. (2018). Relação entre a anteriorização da cabeça, dor muscular e tempo de uso do computador e celular.

Danton, G. (2002). Metodologia científica. Pará de Minas: Virtual Books Online.

de Barros, G. M., dos Anjos, M. S., Mota, D. M., Pessoa, D. R., de Araújo Costa, G., \& Leal, S. S. (2020). Análise dos efeitos imediatos entre a Manipulação Quiroprática e TENS em indivíduos com cervicalgia: ensaio clínico randomizado. Saúde e Desenvolvimento Humano, 8(2), 55-65.

Diehl, A. A., \& Tatim, D. C. (2004). Pesquisa em ciências sociais aplicadas: métodos e técnicas. Pearson Brasil.

dos Santos Pacheco, J., Mejia, D. P. M., \& da Silva Sena, G. G. (2018). Os efeitos do tratamento quiropráxico sobre a cervicalgia: revisão de literatura.

Esteves, B. R., Salvi, L. B., Fagundes, D. J., \& Valverde, P. B. (2013). Effects of chiropractic manipulation on the range of motion of the cervical spine in soccer players. Revista brasileira de quiropraxia, 4(1), 39-49.

Ferreira, V. S., Reis, A. G., Belfort, I. K. P., dos Santos Morais, P. L. T., de Araújo, M. L., de Oliveira Mendes, N., ... \& Barros Filho, A. K. D. (2020). Criação de modelo artificial de avaliação postural para otimização de identificação de desníveis posturais. Revista Eletrônica Acervo Saúde, 12 (4), $3020-3020$.

Fletcher, G. S. (2021). Epidemiologia Clínica-: Elementos Essenciais. Artmed Editora.

Galera, S. R. D. G. P., da Silva, D. A. M., Soares, F. B. M., de Oliveira, P. M., da Silva, A. C. M., de Sales, A. O., \& Porto, R. E. A. (2017). Tratamento da cervicalgia mecânica por meio das técnicas de tração e pompage: relato de caso. Revista Ciência e Saúde On-line, 2(3).

Gama, C. E., Gonçalves, G. B., \& David, R. F. (2019). Efeito da quiropraxia sobre a dor e mobilidade de pacientes com espondiloartrose cervical. Brazilian Journal of Health Review, 2(3), 1773-1787.

Gardin, A. M. V., \& Felipe, F. A. D. A. (2013). Estudo comparativo entre dois protocolos de tratamento Flor de Liz e Método Canal Unitário em algias cervicais. Revista Dor, 14, 290-294.

Gorrell, L. M., Beath, K., \& Engel, R. M. (2016). Manual and instrument applied cervical manipulation for mechanical neck pain: a randomized controlled trial. Journal of manipulative and physiological therapeutics, 39(5), 319-329.

Maia, R. C. B., \& de Freitas, C. F. P. (2012). Técnica Quiroprática: Um estudo teórico sobre seus resultados no tratamento das lombalgias ocupacionais.

Proetti, S. (2018). As pesquisas qualitativa e quantitativa como métodos de investigação científica: Um estudo comparativo e objetivo. Revista Lumen. 2(4).

Silva, R. M. V. D., Lima, M. S. D., Costa, F. H., \& Silva, A. C. D. (2012). Effects of chiropractic care in patients with cervical pain: a systematic review. Revista Dor, 13(1), 71-74. 